\title{
Étudier des chants de funérailles moose
}

Studying Moose Funeral Songs

Moor kvı yıılg valsẽ n gese

\section{Alice Degorce}

\section{OpenEdition}

\section{Journals}

Édition électronique

URL : https://journals.openedition.org/clo/4781

DOI : $10.4000 /$ clo.4781

ISSN : 2266-1816

\section{Éditeur}

INALCO

\section{Édition imprimée}

Date de publication : 21 juin 2018

Pagination : 101-126

ISBN : 9782858313143

ISSN : 0396-891X

\section{Référence électronique}

Alice Degorce, «Étudier des chants de funérailles moose », Cahiers de littérature orale [En ligne], 83 | 2018, mis en ligne le 20 juin 2019, consulté le 01 juillet 2021. URL : http://journals.openedition.org/ clo/4781; DOI : https://doi.org/10.4000/clo.4781

\section{(c) $(1)(9$}

Cahiers de littérature orale est mis à disposition selon les termes de la Licence Creative Commons Attribution - Pas d'Utilisation Commerciale 4.0 International. 


\title{
Étudier des chants de funérailles moose
}

\author{
Alice Degorce \\ Institut des mondes africains (IMAF, UMR 8171 CNRS - 243 IRD, \\ AMU-CNRS-EHESS-EPHE-IRD-Université Paris Panthéon Sorbonne)
}

Dans un article publié dans un numéro spécial des Cahiers de littérature orale intitulé Chanter la mort (1990), Geneviève Calame-Griaule présente un corpus de chants funéraires dogon ${ }^{1}$ qu'elle décrit ainsi après en avoir donné une traduction :

Comme dans tout texte poétique, il s'agit d'une parole énigmatique qui multiplie les images et les formules frappantes, dont le sens immédiat n'est pas toujours évident ${ }^{2}$.

Elle souligne plus loin l'importance de « métaphores poétiques d'une grande force ${ }^{3} \gg$ dans ces textes. Par ces lignes, G. Calame-Griaule pointe un aspect des chants de funérailles qui en font un genre chanté difficile d'accès pour les chercheurs travaillant chez les Dogon et dans les sociétés voisines. En effet, le recours à des procédés stylistiques complexifiant les énoncés autour de métaphores ou d'allusions suppose notamment que l'auditoire ait acquis un certain niveau de connaissance de la langue, de l'art de l'oralité et du contexte local.

1. Ces chants avaient également été publiés dans Ethnologie et langage (CALAME-GRIAULE, 2009), et enregistrés et diffusés sur un disque vinyle (Scènes de la vie des Dogon, 1956).

2. Calame-Griaule, 1990, p. 79.

3.Ibid., p. 84 
Cette contribution a dans cette perspective pour objectif de revenir de façon réflexive sur des questions propres à l'étude de chants funéraires énoncés en moore, la langue des Moose ${ }^{4}$, à partir d'un corpus recueilli en milieu rural au Burkina Faso. Ces chants ont en commun avec la poétique dogon cette importance attribuée à l'allusion, à la manipulation d'images et à la métaphore, ainsi qu'à la présence de marqueurs rythmiques. Leurs paroles intègrent de nombreuses devises individuelles, lignagères, voire de lieux, mêlant ainsi deux genres oraux considérés comme modes d'expression poétique par excellence chez les Dogon, à savoir le chant et les devises ${ }^{5}$. Chez les Moose, l'agencement de devises, de métaphores, de proverbes, ou encore de formules-types propres aux chants funéraires, font que ce genre chanté est considéré comme relevant d'un niveau de langage à la complexité élevée et au style soutenu.

Toutefois, ces références à d'autres genres discursifs oraux et ces procédés stylistiques, tout en faisant la richesse des chants funéraires moose, constituent également un frein pour tout observateur non-initié à leur langage. Les replacer dans leurs contextes d'énonciation et de performance, selon les méthodes inspirées de l'ethnolinguistique ${ }^{6}$ et de l'Ethnography of Speaking and Communication ${ }^{7}$, ouvrent cependant des perspectives d'analyse ${ }^{8}$. Ce contexte doit dès lors être considéré à plusieurs niveaux : celui du contexte social et de l'histoire locale, celui du rituel funéraire et celui de la performance immédiate.

Après une présentation de ces chants de funérailles et de leur contexte d'énonciation, je reviendrai de façon réflexive sur la manière dont l'analyse de ce corpus s'est en partie construite en lien avec ma position de jeune chercheuse sur le terrain. Dans un dernier point, j'analyserai la partie en solo d'une chanteuse de funérailles et, plus précisément, sa construction autour de l'enchaînement de devises, dans ses liens avec l'identité des participants au rite.

Une étude tenant compte de l'histoire locale, de la situation de performance, de l'analyse des relations sociales et du déroulement du rituel permet d'aborder les chants comme faisant partie intégrante du processus funéraire. Le rite a

4. Une partie de ce corpus a été publiée dans la collection Classiques Africains (DEGORCE, 2014). Les Moose, au singulier Moaaga, sont souvent connus sous l'appellation francisée «mossi ».

5. Voir Calame-Griaule, 2009, p. 476.

6. Calame-Griaule, 1970.

7. Hymes, 1962 ; Gumperz \& Hymes, 1964 ; Labov, 1976.

8. Pour des développements plus récents, voir notamment Bornand \& BAUMGardT, 2009 ; BORNAND \& LEgUY, 2013 ; KaPCHAN, 1995. 
en effet pour visée à la fois de séparer un mort des vivants, de le faire devenir ancêtre et de ramener les vivants endeuillés à la vie quotidienne. Les chants funéraires accompagnent ainsi les actes rituels vers ces différents objectifs, allant jusqu'à constituer un moyen d'y parvenir essentiel au déroulement du rite, que seule une approche basée sur l'analyse de la performance et du contexte social d'énonciation permet d'appréhender. Un retour réflexif sur cette approche des chants de funérailles permet ainsi d'aller au-delà des difficultés posées par les procédés stylistiques complexes qui les caractérisent, et de revenir sur leur analyse aux vues des processus sociaux et rituels qui accompagnent l'énonciation des chants dans les funérailles.

\section{L'énonciation des chants dans les funérailles}

\section{Les funérailles et les différents registres de chants}

Le corpus de chants funéraires sur lequel se base cette contribution a été recueilli entre 2003 et 2007 dans un village moaaga situé entre Koudougou et Ouagadougou au Burkina Faso. Le genre chanté spécifique aux funérailles est appelé en moore kvvr yula, dénomination formée sur les termes yulle (au pluriel yula) désignant le chant, et kvvre (pluriel kvya) généralement traduit en français par « funérailles », mais dont le sens pourrait être envisagé de façon plus générale comme ce qui se rapporte à la mort et à son traitement social ${ }^{9}$. Les kvvr yula ont deux principaux modes d'énonciation dans les funérailles. Ils sont en effet chantés soit au cours de veillées funéraires, soit de façon concomitante avec certaines séquences rituelles importantes.

Lorsqu'elles sont célébrées pour un homme ou une femme adulte, marié(e), et décédé(e) dans des conditions qui écartent l'hypothèse d'une mort violente ou prématurée ${ }^{10}$, les funérailles donnent en effet lieu à plusieurs successions de nuits de chants, qui constituent le lieu d'énonciation privilégié des kvvr yula. Les rites qui suivent immédiatement un décès sont ceux de l'inhumation, appelée en moore kv-toogo, expression signifiant «funérailles difficiles »,

9. Degorce, 2014 ; Liberski-Bagnoud, 1989.

10. Selon les discours de nos interlocuteurs, une personne doit être morte « de vieillesse ou de maladie » pour bénéficier des rites funéraires qui vont être décrits, autrement dit, elle ne doit pas avoir subi une mort jugée violente (accident, mort en brousse, assassinat par exemple) ou survenue trop tôt (avant le mariage notamment). 
« qui se font dans la souffrance », « amères ${ }^{11}$ ». Au terme de ces rituels, une première série de veillées est organisée. Leur nombre, censé correspondre au trois masculin et au quatre féminin récurrents dans cette région d'Afrique de l'Ouest ${ }^{12}$ qui supposeraient que trois nuits de veillées soient alors organisées pour un homme et quatre en l'honneur d'une femme, est en réalité déterminé par l'importance sociale du mort et par les moyens dont dispose sa famille, à qui revient la charge de ces premiers rituels. Une longue période de deuil, qui s'étend souvent sur plusieurs années et qui touche principalement les enfants des défunts et leurs conjoints ou conjointes, débute alors. Elle prend fin au moment où la seconde partie des rites est organisée. Celle-ci se doit de signifier aux ancêtres l'importance du mort qui les rejoint, l'accès à l'ancestralité des défunts étant le but des funérailles en tant que rite de passage. Elles sont aussi l'occasion de démontrer aux vivants le prestige de la famille d'un ou d'une défunte, mesuré par la capacité à organiser des rites grandioses.

De nouvelles nuits de veillées funéraires, au cours desquelles des chants kvor yula sont encore entonnés, sont alors organisées quelques jours avant et après cette deuxième partie des rites. Pour un homme, elle se divise en deux phases, qui se tiennent généralement l'une à la suite de l'autre. La première d'entre elles est appelée kv-landgo, expression traduite ici par «funérailles de réjouissances » à partir des propos de mes interlocuteurs. Les « funérailles de réjouissances » constituent une séquence du rite qui doit manifester la grandeur d'un mort, non seulement de par une atmosphère générale festive « obligatoire ${ }^{13}$ », mais aussi par l'importance des sacrifices offerts par les enfants des défunts et par leurs gendres. La dernière partie du rite est organisée immédiatement après, et porte pour nom kv-yiibu (sortir les funérailles) ou kv-yiisgu (faire sortir les funérailles). Elle concerne la levée de deuil des orphelins et des veuves ou veufs, et marque simultanément le départ du mort dans l'au-delà.

Le terme kvore est employé pour désigner l'ensemble des secondes funérailles d'une femme. Les différentes étapes rituelles exécutées pour un homme se retrouvent dans les rites féminins, mais leur désignation ne fait pas nécessairement appel à l'ensemble des appellations rapportées ci-dessus. Les funérailles des femmes comportent en revanche une ultime étape qui leur est spécifique et qui concerne le retour de l'esprit de la défunte dans son lignage

11. Norbert \& Kinda, 1997.

12. FAINZANG, 1985.

13. L'usage de cette expression fait ici référence au titre de Marcel Mauss : « L'expression obligatoire des sentiments », MaUss, 1968. 
d'origine. Cette phase est appelée $k v$-peoogo, expression de nouveau formée du radical $k v$ - (kvvre), auquel est ajouté le substantif peoogo [panier], qui désigne ici une grande corbeille dans laquelle sont rassemblées ses affaires personnelles et quelques dons d'argent ou de noix de kola, qui est considérée comme le support de son esprit que l'on ramène « chez elle ».

À l'inverse des performances nocturnes des chants au cours des veillées qui rythment les différentes étapes du processus funéraire, leur seconde situation d'énonciation est diurne et se fait de façon parallèle à des gestes rituels importants. Les interprètes vont alors prendre place non loin de l'endroit où sont exécutés les actes rituels, pour y entonner leurs chants dans un espace proche mais bien distinct de celui de la scène rituelle. Au cours de ces performances diurnes dans le rite, les parties en solo sont courtes et la répétition de refrains prend plus d'importance.

D'autres genres chantés coexistent avec les chants kvor yula dans les funérailles : des chants de jeunes filles, des chants entonnés par les gendres des défunts et des chants de masques. Les chants de jeunes filles sont des chants de la période prénuptiale, habituellement chantés les soirs de saison sèche, qui sont repris au cours des funérailles par les belles-filles des défunts ou des défuntes ${ }^{14}$. Les chants des époux des filles des morts sont des chants d'amour chantés pendant les « funérailles de réjouissances » d'un homme ou les secondes funérailles d'une femme : leur énonciation, qui va de pair avec les offrandes des gendres adressées aux défunts pour leur départ dans le monde des ancêtres, participe à l'atmosphère sonore festive qui doit émaner des funérailles et donc à signifier l'importance des futurs ancêtres. Enfin, les chants de masques ne sont énoncés qu'à l'occasion des funérailles célébrées dans des lignages de propriétaires de masques appelés sukoomse. Ces masques s'expriment par leurs danses, qu' ils exécutent devant trois longs tambours couchés au sol et un chanteur qui s'exprime dans un langage initiatique $^{15}$.

\section{Énonciation des chants de funérailles lors des veillées}

Le corpus sur lequel se base cet article a essentiellement été recueilli au cours de veillées de secondes funérailles. Les veillées peuvent être appelées wama ([calebasses], pluriel de wamde) dans certaines localités de la région de Koudougou, en raison du fait que des calebasses coupées en deux et retournées sur

14. DEgORCE, 2016a.

15. Ces chants et ces danses sont entourés de nombreux interdits, et ne font pas l'objet de nos travaux. 
des couvertures comptent parmi les principaux instruments qui accompagnent les chants. La dernière nuit, celle qui précède la levée de deuil, est appelée wam lokre [calebasse/détacher, libérer], expression incluant une idée de dénouement. Alors que les veillées qui précèdent celle-ci peuvent s'achever au cours de la nuit, après plusieurs heures de chants, la veillée wam lokre doit durer jusqu'au lever du jour.

Les épouses des fils d'un défunt ou d'une défunte sont toujours les premières à chanter. Elles sont souvent relayées par d'autres chanteurs ou chanteuses et, si le défunt est important, d'autres groupes musicaux peuvent s'installer autour de la cour. L'interprétation des kvvr yula s'organise autour de duos de chanteuses, composés d'une soliste et d'une seconde voix qui l'accompagne par des vocalises. Les deux interprètes se tiennent debout et des couvertures sur lesquels sont disposés les instruments de musique sont posées devant elles. Ces instruments sont essentiellement les demi-calebasses retournées dont il vient d'être question, et un ou deux tambours d'eau appelés ko-libri [eau/retourner], composés d'un grand récipient en terre rempli d'eau dans lequel flotte une petite moitié de calebasse retournée qui est frappée par les musiciennes avec une louche, également découpée dans une calebasse. Ces tambours d'eau sont les instruments des veillées par excellence et, qui plus est, les instruments des femmes : ils sont en effet joués presqu'exclusivement par ces dernières et sont associés au deuil, ne pouvant être frappés qu'entre le décès d'un individu et la levée de deuil de ses proches ${ }^{16}$. Enfin, des danseurs se suivent en file indienne autour des tambours et des duos de chanteuses, reprenant en chœur les refrains lancés par les solistes.

Les chants sont en effet composés selon un mode responsorial, où un chœur répond à une ou à un soliste. Ces allers et retours entre le soliste et le chœur sont accompagnés par les vocalises d'une seconde soliste. Ces vocalises, quoiqu'apparaissant secondaires, concourent à inculquer à ces chants une dimension émotionnelle particulière.

\section{Images, métaphores et rythmique des chants funéraires}

L'aspect émotif est d'autant plus appuyé dans les chants parlant directement de la mort ou du départ des défunts. Toutefois, les thèmes abordés par les chanteurs ne se limitent pas à ce registre, pouvant également traiter de problématiques du quotidien, parfois sur un ton humoristique. Entre leurs refrains, les solistes

16. Degorce, 2014. Si les musiques et les chants se prolongent au-delà de la levée de deuil, ils seront remplacés par d'autres instruments, par exemple fabriqués avec des bidons en plastique. 
peuvent insérer des parties plus libres. Ces solos sont en grande partie composés d'un « stock » de formules et d'énoncés agencés par les solistes selon les circonstances de l'énonciation ou leurs propres personnalités : références à la mort, au jeu des tambourinaires, au chant de l'accompagnatrice ou de l'accompagnateur, aux lignages du mort et de ses alliés, à ceux des membres de l'auditoire, formules spécifiques aux chants de funérailles, discours proverbial, chansons entendues à la radio sont autant de répertoires dont les chanteuses et chanteurs s'inspirent pour leurs parties en solo. L'allusion, la métaphore ou l'ellipse sont abondamment utilisées, tant pour construire ces parties en solo à partir de ces différents types d'énoncés que dans les refrains.

Les deux refrains suivants illustrent cet usage de la métaphore. Le premier met en garde les jeunes filles trop aventureuses contre les grossesses non-désirées, faisant allusion au ventre d'une femme enceinte en l'appelant le « fruit rond du calebassier du chef Wẽnde ${ }^{17} \gg$. Le second porte sur la mort, comparée à une puisette venant salir l'eau d'un puits.

Écoutez, une femme qui est tombée enceinte

Va voir le monde, et tombe enceinte

Sait renvoyer la parole (répondre), et tombe enceinte

Va voir le monde, et tombe enceinte

Petits-enfants oh, et tombe enceinte

C'est le fruit rond du calebassier du chef Wênde comme ça

C'est l'amour du mari qui a eu la force comme ça

Femme yeh

Kelge pag sẽn tvta pug we

Na n yãa Moogo n tvta pvgyaa

Mii gomd lebg n tvta pugyaa

Na yãa Moog n tvta pug yaa

Kombi yoo n tvta pug yaa

Yaa naaba Wênd kãan-gilg la woto

Yaa sıd nonglm paam pãng la woto

Pagyee

17. Wẽnde est le nom de la divinité principale des Moose, et désigne également Dieu pour les catholiques et les chrétiens évangéliques. 
Ne gâteront pas

Cent puisettes tombées dans le puits ne gâtent pas l'eau

L'eau est propre

Kõn sãam ye

Luvd koabg lvu bulgẽ pa sãam koom ye

Koomã yaa yılemde

Ce dernier chant dit ainsi de la mort qu'elle ne pourra toucher toute la communauté des vivants, tout comme des puisettes tombées dans un puits, même nombreuses, ne pourront en salir l'eau.

Aux côtés de l'usage des images métaphoriques ou allusives, G. Calame-Griaule remarque dans Ethnologie et langage l'importance des marqueurs rythmiques dans le discours poétique dogon. Cette caractéristique n'est pas étrangère aux chants de funérailles moose. Dans l'extrait ci-dessus parlant des grossesses non désirées, la chanteuse rythme ses vers (du deuxième au quatrième vers) en les terminant à chaque fois par « et tombe enceinte », juxtaposant finalement deux énoncés en un seul vers ${ }^{18}$ et les faisant répondre au premier, qui se terminait par « ...qui est tombée enceinte ». Les chanteurs des funérailles font régulièrement appel à ce procédé, notamment dans leurs parties en solo. Le solo détaillé dans la dernière partie de ce texte en est également une illustration, la chanteuse donnant une forme d'écho d'un groupe de vers à l'autre en utilisant successivement comme marqueurs rythmiques des parties provenant d'autres vers (《n'est pas là », « c'est une femme », « c'est un sac oh ») ou des sonorités particulières («yeb oh »). G. Calame-Griaule note cette importance du rythme qui participe à donner aux paroles des chanteurs dogon (ou moose) leur impact : «Il s'agit-là d'un procédé nettement stylistique dont le récitant peut jouer avec plus ou moins de maîtrise à partir d'un certain nombre de formules types. Le texte en acquiert une force et un rythme frappants ${ }^{19}$. 》

Tout en constituant un élément prépondérant des chants et en conférant sa richesse au genre, l'usage abondant de ces différents procédés en complique la compréhension pour l'auditeur novice. C'est précisément autour de cette complexité qu'une position réflexive semble devoir s'articuler avec l'apport

18. Séparés dans la transcription en moore par la particule de liaison «n $n$ et dans la traduction en français par des virgules.

19. Calame-Griaule, 2009, p. 481. 
méthodologique des recherches sur la situation d'énonciation et sur la performance.

\section{Retour sur un terrain}

Ces travaux sur les chants funéraires moose ont été effectués dans le cadre d'une thèse, et ont ainsi coïncidé avec un premier apprentissage de la langue moore. Cette thèse avait dans un premier temps pour thème les rituels funéraires moose. Toutefois, j'ai rapidement été renvoyée à l'étude des chants de funérailles.

$\mathrm{Au}$ cours de ce terrain en effet, et en raison de mon âge au moment des enquêtes, je me vis rapidement assimilée au statut des jeunes épouses, statut auquel je n'étais toutefois pas assignée. Du fait de mon altérité et de mon extériorité à la société, il m'était cependant possible de m'en extraire et de mener par exemple des entretiens avec des hommes âgés. Cette liberté de circuler d'un statut à l'autre avait néanmoins des limites concernant notamment certains rituels sacrificiels, ou encore les danseurs de masques.

Mes interlocuteurs m'ont ainsi conduite de façon indirecte à me pencher sur les rôles rituels des jeunes épouses des enfants des défunts. L'un de ces principaux rôles consistait en l'énonciation des chants de funérailles, qu'elles sont systématiquement les premières à entonner dans les veillées.

J'ai alors pu enregistrer plusieurs heures de chants lors de différentes veillées funéraires. Mais plusieurs difficultés se sont posées lors du travail de transcription et de traduction de ces chants, pour lesquelles j'ai travaillé avec plusieurs locuteurs de la langue. La première résidait dans les références ou allusions très locales (aux lignages ou à l'histoire du village par exemple) des chants, difficilement compréhensibles pour les personnes extérieures à la localité. Les extraits suivants mettent par exemple en scène deux personnages liés à l'histoire du village. Le premier est Rabi, arrière-grand-père maternel d'un des chanteurs, issu d'un des quartiers du village où j'ai mené l'essentiel de mes enquêtes. Ce refrain était entonné dans la plupart des veillées, s'adressant aux descendants de Rabi (soit parce que les funérailles se tenaient dans leur lignage, soit en guise de salutations ou d'hommage s'ils assistaient à une veillée). Rabi était connu pour avoir pris pour épouse une femme venant d'un village situé à l'Est. Le second personnage est Emprunteur-de-Chien, un homme du village connu pour sa conduite jugée asociale. À la mort de son chien, celui-ci aurait en effet plus pleuré qu'il ne l'aurait fait pour un être humain. Ici encore, la référence est locale et peu, voire pas, connue en dehors du village. 
Ici, c'est l'Est

Rabi, oh

Ici, c'est l'Est

Ka la yaang yee

Rabi woo

Ka la yaang yee

Je pleure $m$ ba $y e^{20} \mathrm{eh}$, eh, eh (bis)

L'homme Emprunteur-de-Chien pleure $m$ ba yé

Il n'aura plus de chien pour entrer en brousse

Emprunteur-de-Chien

Oh, je pleure $m$ ba yé

Mkumda $m$ ba yee e e e (bis)

A raoa Ba-Reggdkumda ba yaa

Pa na leb $n$ yã baag $n$ kẽ weoogo

A Ba-Rergdo

Woo $m$ kumda $m$ ba yee

Une autre difficulté résidait dans le fait que les entretiens avec les chanteuses et chanteurs trouvaient rapidement leurs limites. Ceux-ci développaient en effet peu de discours explicatifs autour de leurs chants. Souvent, les chanteuses esquivaient les questions ou répondaient a minima, prétextant parfois leur ignorance, et les hommes les évitaient en faisant de longues digressions. Quelques informations au cours de ces entretiens menés a posteriori des veillées ont néanmoins pu être obtenues mais elles étaient loin d'être satisfaisantes. Une première conclusion a alors été qu'il était difficile pour ces chanteuses et chanteurs de revenir sur des discours énoncés dans un contexte performatif très particulier, dans lequel les interactions avec l'auditoire sont incontournables. Toutefois, cette explication n'était pas le propre de ces chants de funérailles et pouvait au contraire caractériser la plupart de ce type de performances orales.

Une autre explication à propos de cette difficulté à mener des entretiens autour des chants de funérailles, et notamment lorsqu'il s'agissait d'entrer dans le détail de leurs paroles, pouvait résider dans le fait que le langage des chants

20. Idéophone exprimant les pleurs et les cris de deuil. 
de funérailles transgresse les normes de la parole quotidienne. Cette spécificité du discours funéraire chanté est exprimée dans les paroles des chanteurs. Ceux-ci s'entourent en effet de précautions avant d'entamer leurs tours de chants, en s'excusant et en demandant la permission de chanter par exemple. Ils emploient alors une expression synonyme de «demander la permission » dans des chants d'ouverture du type de celui-ci :

Pardon gens du hangar oh

Je vais pleurer $m$ ba ye

Pardon yeh eh ah eh

Je demande la place [la permission]

Pour pleurer mon père et ma mère, oh

Kabr yee zãande dãmba woo

$M$ na n yäb mam bae

Kabryee ee ae

$M$ kota weere

Nyãb mam ba ne m ma woo

Les « gens du hangar » sont ici les aînés du lignage qui dirigent la cérémonie, assis sous un hangar érigé devant la cour du défunt ou de la défunte. Le corpus de chants funéraires dogon recueilli par G. Calame-Griaule commence également par un chant d'ouverture de ce type, dont elle rappelle l'importance dans le contexte de rites funéraires :

Ce premier chant est une formule d'ouverture qui joue un rôle propitiatoire. Tout rituel de parole (chant religieux, prière...) commence en effet par une demande de pardon aux ancêtres et aux puissances tutélaires pour éviter que les paroles prononcées ne les offensent. Cette valeur propitiatoire est d'autant plus importante qu'il s'agit de chants de mort et qu'on ne saurait manier avec trop de précautions tout ce qui la concerne : la mort est facilement contagieuse $^{21}$.

Dans leurs solos, les chanteurs, mais surtout les chanteuses des funérailles moose (ces vers ont été plus souvent retrouvés dans les répertoires féminins de ce corpus), introduisent par ailleurs les formules suivantes, qui traduisent d'une certaine manière leur transgression des normes langagières quotidiennes. Elles

21. Calame-Griaule, 1990, p. 80. 
entrent de ce fait dans une forme de discours chanté prescrite par le rite ${ }^{22}$, où la parole adopte une volubilité peu valorisée au quotidien :

On dit que j'ai une grande bouche

Ma bouche-là a commencé tôt

J'ai commencé à parler tôt, quand j'étais petite

Je ne vais pas avoir honte de ma bouche

C'est mon père qui m'a fait naître avec la parole !

Yet $m$ bee noor zañ yee

$M$ noor yónog singa pind yee

$M$ singa pind t'm gomd t' $m$ paoode yee

Mam kõn zoe $m$ noor yãnd ye

$M$ ba $n$ dog $m$ ne gomd yoo, kato!

Les chants funéraires rappellent ainsi eux-mêmes les limites de la parole de leurs interprètes, et la spécificité de celle-ci, spécificité liée au contexte funéraire et rituel qui la rend difficilement maniable ou qu'on ne peut même aisément commenter en dehors de sa situation d'énonciation. Ce genre oral n'est par ailleurs pas chanté en dehors du contexte des funérailles : il peut être enregistré et diffusé sur différents supports audiovisuels, mais ne sera pas entonné en dehors de sa situation de performance dans le rite.

Malgré ces limites posées par le genre et par ses conditions de performances, plusieurs entretiens ont pu être menés auprès d'hommes âgés qui expliquaient quant à eux volontiers les textes des chants. D'une part, ils avaient une longue pratique d'écoute des chants lors des veillées et ils connaissaient également très bien l'histoire du village et des familles, les allusions des chants, les devises des lignages. Mais d'autre part, du fait de leur statut d'aînés masculins, ils étaient également autorisés à me délivrer certaines informations nécessaires à la compréhension des chants ou même des rites funéraires.

L'observation des situations de performance des chants de funérailles a permis de compléter les informations obtenues par l'intermédiaire de cette « parole autorisée » des aînés. Cette démarche a conduit à interroger non seulement l'identité des participants aux veillées (duos de solistes, chœur et auditoire), mais aussi celle des personnes et des lignages impliqués dans la célébration des funérailles, du fait de l'importance de l'énonciation de devises, notamment de devises lignagères, dans les chants de funérailles. 


\section{La transformation des morts en ancêtres et l'énonciation des relations sociales}

Les rites funéraires marquent la rupture avec les défunts par toute une série de gestes rituels et de manipulations d'objets symboliques ou de représentations des morts (par exemple, la natte qui a entouré le cadavre juste après le décès qui est employée à nouveau lorsque l'inhumation est rejouée, l'arc et le carquois d'un homme ou le panier de mariage d'une femme). Les chants accompagnent ce processus en rappelant tout d'abord que les conditions du décès et le statut social permettent à un défunt d'accéder au statut d'ancêtre. Dans leurs parties en solo, les chanteurs peuvent ainsi évoquer le fait que le défunt est parti d'une « bonne mort », énumérant par exemple les situations évitées. Ils font aussi allusion au fait que le défunt était marié, qu'il n'avait pas de dette ou n'avait pas connu la honte ${ }^{23}$.

Les chants véhiculent ainsi des images du défunt qui le rendent conforme à la représentation d'un mort « ancestralisable ». L'un des procédés les plus couramment retrouvés d'un corpus à l'autre est l'usage répété de surnoms qui permettent aux chanteurs d'attribuer aux morts les qualités attendues d'un ou d'une aînée. Ces surnoms sont par exemple souvent introduits en préambule à une formule typique des chants de funérailles qui se termine à chaque fois par menma Moogo [a disparu du monde], et peuvent ainsi avoir trait à la parole des anciens, porteuse de vérité ou de conseils avisés ${ }^{24}$ :

Appelle-et-Conseille a disparu du Moogo [du monde]

Bool-n-Sagl menma Moogo

\section{Dit la Vérité a disparu du Moogo A Sid-Yeta menma Moogo}

Ici encore, la répétition de ces formules participe à la rythmique des chants, qui sont également marqués, notamment dans leurs parties en solo, par la présence de nombreuses devises. De façon générale, le sens des devises est particulièrement difficile d'accès : tout comme les devises dogon, l'image y est importante, par un nouvel usage abondant de métaphores ${ }^{25}$. La connaissance

23. Degorce, 2016b.

24. Degorce, 2016b; Degorce, 2014.

25. Calame-Griaule, 2009, p. 483. 
$\mathrm{du}$ contexte s'avère alors essentielle pour l'auditoire, afin de savoir de quelle personne, de quel lignage ou même de quel lieu on parle.

Les devises concernant les personnes sont de deux principales sortes. Les noms de combat [zab-yvya] tout d'abord sont des devises qu'un individu se donne lui-même, généralement à l'âge adulte, pour affirmer et clamer certains de ses traits de caractère, ou pour s'exprimer à propos d'une situation particulière, souvent conflictuelle ${ }^{26}$. Il s'agit donc là d'un nom choisi par son porteur. Les chefs moose arborent ainsi une devise de ce type au moment de leur intronisation, dont un terme sera leur nom de chef [naaba]. Chaque individu, homme ou femme, selon les circonstances et les événements qu'il traverse, peut s'attribuer un ou plusieurs zab-yvvre [nom de combat]. Ces devises sont intégrées dans les parties solos des chants de veillées, qu'il s'agisse de celles du mort lui-même ou d'autres aïeux disparus. La personne se l'étant attribuée elle-même, ce nom de combat est révélateur d'un trait de caractère qu'elle tient à mettre en avant, souvent pour mettre en garde ou provoquer ses ennemis, ou pour faire son propre éloge, dans le but de faire impression.

Cependant, ces devises ne peuvent révéler leur sens et donner des informations sur des traits personnels de leurs porteurs au-delà de leur mort qu'à condition que quelqu'un ait gardé la mémoire du contexte dans lequel elles ont été élaborées. Elles ont en effet une valeur de message et, allusives et métaphoriques, leur fonction générale consiste à mettre en valeur un trait de la personnalité de leur porteur, qui en est donc à la fois l'auteur. La signification de celles qui sont citées dans les chants funéraires devient souvent assez obscure pour les plus jeunes qui n'ont connu ni celui qu'elles concernent, ni le contexte dans lequel elles ont été déterminées.

Les devises lignagères sont appelées soanda (au singulier, sondre). L'énonciation de ce type de devises permet aux descendants d'un lignage de se situer par rapport à l'histoire du village, aux différentes familles qui le composent, mais aussi de se référer aux valeurs fondatrices qui sont véhiculées dans ces devises. Comme le signale G. Calame-Griaule, la récitation des devises d'un défunt peut aussi être une façon de le remercier pour les bienfaits accomplis de son vivant ${ }^{27}$.

Le découpage du chant qui suit vise à analyser à partir de la partie en solo d'une chanteuse, une épouse qui chante lors des funérailles de sa belle-mère, la façon dont l'étude de la situation d'énonciation des devises dans les chants de funérailles informe sur les liens entre les participants aux funérailles et aux

26. Sur les zab-yvya [noms de combat], voir notamment les travaux de KaWAdA, 1998.

27. Calame-Griaule, 2009, p. 372. 
veillées. Il s'agit de comprendre à qui fait référence et à qui s'adresse la succession de devises que l'interprète introduit ici, grâce à l'introduction d'annotations tout au long du chant.

Répétition d'une phrase du refrain (marque le début du solo):

Que faire de macaronis après son départ yeh

Que faire de macaronis après son départ yeh

Macaronis eh eh

Yõdg n maana makoro yee

Yödg n maana makoro yee

Makoree

Devises [soanda] du lignage paternel de la défunte :

«Enlève ce qui n'est pas doux » n'est pas à la maison

Et argumente pour se laver demain

Et amène-moi pour que j'atteigne et vienne

Et les enfants de « Butte de terre et œufs de margouillats » oh, oh

[Vers inaudible]

Le long tambour en a eu un, oh, oh

Ce sont les gens de l'Est qui sont longs yeh, n'est pas là eh

La longueur ne surprendra pas de nouveau la vipère yeh, n'est pas

Le vent de Ba a pris la vie yeh, n'est pas là eh

«Pleurer le Couur » de Kindi yeh, n'est pas là eh

Pa noomã rokom ka yir

La padm koom soodbeoogo

La tall-ma to $m$ ta $n$ wa

La kunkubr n bãng gezl koamba woo o

[Vers inaudible]

Gãngãoog wok paamd a yembr woo o

Yaanga rãmb la woglem yee ka yee

A woglem le kón ling robg yee ka ye e

T'a Ba sebgã rlk yõor yee ye ka ye e

T'a Kindi Kum Sünr yee ka yee

Vers de transition :

Mais quelle brousse ont-ils quittée ?

Dans quelle brousse vais-je repartir, oh ? 


\section{La bãmb yala yeznẽ weoog yee?}

Mam na n leba yeznẽ weoogo wo?

« Nom de combat » de la défunte :

Tampokien de Pibin ${ }^{28}$, c'est une femme

Et dit qu'une femme ne se courbe pas, c'est une femme

Elles ont refusé de se courber, zole yah

Elle dit la tortue n'est pas la bouche, zole yah

[Vers inaudible]

A Pibẽ Tampokié yaa paga

Nyeti pag ra zõond yaa paga

Tı bämb tôdg n zôond zol yaa

Yeta kur pa noor zol yaa

[Vers inaudible]

Références au village d'origine de la défunte :

Et dit rencontrer Tamissin ${ }^{29}$, zole yeh

Et s'occuper de la gauche, zole yeh ${ }^{30}$

Nyeti seg Tamissẽ zol yee

$N$ zã goabga zol yee

Vers de transition :

Je veux saluer mon hier [pour le bienfait d'hier] et rentrer chez

En vérité, a quitté quelle brousse ?

$M$ data pvos m zaamẽ la kul yee

Sid yıla yesnẽ weoogo?

Devises du lignage paternel de la seconde voix (lignage de forgerons):

28. Quartier où réside le lignage paternel de la défunte.

29. Village d'origine du lignage paternel de la défunte.

30. Les marqueurs rythmiques (zol yee/zole yeh) permettent une continuité avec l'énonciation du nom de combat de la défunte. 
C'est le grand-père des forgerons « Libérer le monde »

C'est « Démonter la houe et rembourser la dette », oh oh Souffle, j'arrive demain, yeb oh

La femme chérie appelle la forge, yeb oh

Et pourtant elle n'attachera pas pour détacher, yeb oh

Le bord du marigot a refusé la pluie, yeb oh

Yaa sãab yaaba Baagr yee Moogo

Yaa baag wãk $n$ rond samde woo o

A zugdê tvm waat beoogo yeb woo

Pvg-nongre boond kudgu yeb woo

A yaool $n$ kõn sẽneg $n$ yaoongo yeb woo

A bãoog noor tốd saag yeb woo

Vers de transition :

Dans quelle brousse allons-nous repartir, oh ?

Tônd na n leba yesnê weoogo woo ?

Devises du lignage paternel de la soliste :

Le bendre ${ }^{31}$ du chef de Zaktogo ${ }^{32}$, eh

«Le bendre ne frappera pas le masque »

Le bendre de «Ne frappera pas le masque », c'est elle

Le froid de Bingo ${ }^{33}$ amadoue le vent

Il souffle et rafraîchit l'endroit

Dit « C'est la gauche qui n'est rien »

Elle suit et flatte la femme oh [elle - la gauche - a pu flatter une femme]

A Zagtog naaba bendre e

A bendr kôn ting waongo

$A$ kôn tĩng waongo bendre yaa yẽnda

A Bing waood belemd sebgyee

T'a fugda $n$ maag zĩiga

31. Nom d'un tambour moaaga, notamment utilisé pour chanter les devises des chefs.

32. Quartier où réside le lignage paternel de la soliste.

33. Village d'origine du lignage paternel de la soliste. 
Vers de transition :

Dans quelle brousse allons-nous repartir, oh ?

Tönd na n leba yeznẽ weoogo woo?

Formules de chants funéraires (rôle de la première épouse) :

En réalité, c'est la femme-aînée qui possède la maison

Elle possède la maison mais n'égorgera pas un poulet

Mik pug-kẽem n so yir

A so yir kõn kodg noaag

Vers de transition :

Quelle brousse vais-je quitter, eh eh ?

Mam na n yala yeznẽ weooge e ?

Devises du lignage paternel de la troisième soliste :

Je m'excuse encore, Balinga ${ }^{34}$ de Soulou ${ }^{35}$ oh

Le granit gluant est tombé

L'étranger apparu brusquement ramasse

[Vers inaudible]

Le Balinga bat [avec] son marteau

Mam ye kabsa Sulu lingu woo

A pii saoolgo lvı yerre

A sãana poogr n nogde

[Vers inaudible]

Balinga zabda a gũngu

Vers de transition :

Je vais retourner où, eh eh ?

Mam na n leba yernẽ e e ?

Devises du lignage paternel de la troisième soliste (suite) :

Le chef Rali de Poé ${ }^{36}$, c'est un sac oh

34. Nom d'un masque.

35. Nom d'un quartier d'un village voisin.

36. Nom du village d'où est originaire la troisième soliste de la veillée. 
Il s'est adossé au tamarinier pour juger, c'est un sac oh La bonne chèvre $B u-z a m a^{37}$ a eu ma peau, c'est un sac oh

Celui pour qui c'est interdit est assis avec un sac poilu, c'est un sac

T'a Poee naaba Rali yaa korgo woo

A rel pusg kaood bvvdo yaa korgo woo

Bv-zam sõng tar m gãong yaa korgo woo

A kis medgãzĩin ne kombdo yaa korgo woo

Vers de transition :

Dans quelle brousse vais-je retourner, oh ?

Je salue mon hier [pour le bienfait d'hier] et je rentre chez moi

Mam na n leba yesnẽ weoogo woo?

M povs $m$ zaamẽ la $m$ kul yee

Devises des Peuls (en référence aux habitants d'un quartier silmi-moaaga ${ }^{38}$ voisin) :

La tresse unique a obtenu, Peul

Les enfants de la maison de « Vagabonde en brousse », Peul eh

Gem a yembr paama yaa wulma

A saad moog yir koamba yaa wulma e

Répétition d'un vers du refrain :

Macaronis eh eh

Que feras-tu de macaronis après son départ ?

Faire de macaronis après son départ $(\mathrm{x} 4)$

Makoree

Fo na yõdg n maana makoro yee?

Yödg n maana makoro yee

37. Espèce de chèvre.

38. Lignage issu de l'union prohibé entre Peuls et Moose. 
Devises du lignage paternel de la défunte :

J'ai appelé le Zoaaga ${ }^{39}$ de Pibin ${ }^{40}$ [fin du vers inaudible], c'est une femme

[Vers inaudible]

La tortue est entrée dans la jarre, c'est une femme

M bool a Pibẽ Zoaaga [fin du vers inaudible]

yaa paga

[Vers inaudible]

Kur kẽ yvurẽ yaa paga

Interpellation des membres du lignage du mari de la défunte :

J'ai appelé le chef de Tanghin ${ }^{41}$

[Vers inaudible]

Les gens de Zoaaga ${ }^{42}$ de Tanghin ne sont-ils pas à côté ?

M bool a Tãngẽ naab yee

[Vers inaudible]

Tãngẽ Zoaaga rãmb ka yamba la?

Marquage de la fin du solo :

Yeh eh eh eh

Yeh eh eh eh

Yee e e

Yee ee

Le terme weoogo, qui désigne au sens premier la brousse, peut également être employé pour parler d'une région voire, dans ce cas précis, d'un quartier. Articulant le passage d'un groupe de devises à l'autre par des questions du type «Dans quelle brousse vais-je repartir ? », appelées plus haut « vers de

39. Nom d'un masque.

40. Quartier où réside le lignage paternel de la défunte.

41. Nom du quartier où réside le lignage du mari de la défunte.

42. Nom d'un masque. 
transition », la chanteuse crée au long de son récital un véritable cheminement au travers des lignages et des quartiers mobilisés par les funérailles.

Les devises du lignage paternel de la défunte, fondateur d'un quartier relativement éloigné, sont tout d'abord citées. Cet extrait a été recueilli au cours de la première veillée des secondes funérailles de la vieille femme, célébrées chez son mari ${ }^{43}$. Ses paternels n'assistent pas à cette première nuit de chants, ils n'arriveront en effet que le surlendemain : leur énonciation a ainsi suscité moins de réactions parmi l'assistance que celles qui accompagneront la suite du chant.

Par la citation du « nom de combat » de la morte ensuite, la soliste l'évoque directement et des cris l'encourageant à aller de l'avant émanant de son auditoire ne se sont pas fait attendre. Les deux séries de devises suivantes concernent les lignages paternels de la chanteuse elle-même et de son accompagnatrice, également mariée à un fils de la défunte. Les chanteurs évoquent ainsi très souvent leurs propres devises lignagères, non pas pour s'auto-louanger mais pour appeler les membres de leurs lignages à les soutenir dans leurs performances.

Les deux vers qui suivent sont des formules spécifiques aux kvvr yula (chants $\mathrm{du}$ kvvre) rappelant les prérogatives de la première épouse d'un doyen de lignage, statut qu'a eu à occuper la défunte du vivant de son époux : doyenne de la cour, elle a ses coépouses sous sa responsabilité, ordonne la vie quotidienne de la maisonnée et joue un rôle indispensable dans les différents rituels sacrificatoires du lignage, qui ne peuvent avoir lieu sans sa présence, bien qu'elle ne soit traditionnellement pas autorisée à égorger un animal.

Les devises suivantes sont ensuite celles du lignage paternel de la troisième soliste, puis d'habitants d'un quartier voisin qui participaient à la veillée. Enfin, celles des paternels de la défunte sont rappelées avant que ne soient énoncées celles du lignage de son époux, les deux lignages alliés matrimoniaux responsables de la conduite des rites funéraires étant associés dans cette partie du chant.

Ces énonciations des devises lignagères des défunts dans les chants funéraires permettent tout d'abord de replacer les morts par rapport aux lignées ancestrales qu'ils rejoignent. Mais elles situent également les membres de leurs familles par rapport à leurs lignages et à leurs histoires. Par l'énonciation des devises des lignages des autres participants à la veillée, les morts et leurs descendants sont situés par rapport au réseau de parenté et d'alliances qui structurent le village. Avec l'évocation de ces liens intra- et inter-lignagers, la position du mort pour qui l'on chante est donc à la fois spécifiée et inscrite au sein de la communauté. Il en est de même pour les vivants, les chants funéraires jouant par ailleurs un

43. La résidence étant patrilocale, il s'agit aussi du lieu où elle vivait. 
véritable rôle de transmission de cette histoire des réseaux relationnels locaux vis-à-vis des jeunes qui assistent aux veillées.

\section{Conclusion}

Cette contribution avait donc pour objectif de présenter comment, à la nécessité théorique de prendre en compte la situation d'énonciation ou de performance des chants funéraires, s'est adjointe une démarche allant en ce sens impulsée sur le terrain et par les spécificités de cet objet de recherche que constituent les chants de funérailles. Un autre objectif était de lier cette réflexion à l'œuvre de G. Calame-Griaule : d'une part parce que ses recherches ont figuré parmi celles qui ont suscité les développements actuels des travaux allant de le sens de l'étude de la performance, et d'autre part, pour revenir sur cet article sur les chants de funérailles dogon (quoique plus marginal dans son œuvre), et sur ses réflexions sur l'art de la parole dogon (Ethnologie et langage).

Ces différents éléments ont été essentiels dans la construction d'une réflexion sur le rôle des chants de funérailles moose dans le processus de deuil, tant vis-à-vis des morts et de leur transformation en ancêtres que des vivants, de leurs réseaux de relations et de l'énonciation de celles-ci dans les chants de funérailles. Chaque chanteur compose ainsi son discours en fonction de son assistance, laquelle est à chaque fois différente et déterminée par l'identité sociale et par l'histoire des relations d'un défunt. Chacune de ces performances, tout en répondant à des règles d'exécution précises, voit ainsi sa coloration étroitement liée au réseau relationnel dans lequel était inséré un mort. La connaissance du contexte et de ces réseaux de relations s'avère donc essentielle pour la compréhension des chants, constituant une porte d'entrée incontournable pour aller au-delà de la complexité conférée par leurs procédés stylistiques et leur poétique.

\section{Bibliographie}

Alexandre Gustave, 1953, La langue Möré, 2 tomes, Sénégal, 407+506 p.

Andreesco Ioana (dir.), 1990, Chanter la mort, Cahiers de littérature orale, $\mathrm{n}^{\circ} 27$.

Baumgardt Ursula \& Bornand Sandra (dir.), 2009, Autour de la performance, Cabiers de littérature orale, $\mathrm{n}^{\circ} 65$. 
Bornand Sandra \& Leguy Cécile, 2013, Anthropologie des pratiques langagières, Armand Colin (coll. Collection U. Sciences humaines \& sociales), Paris, $205 \mathrm{p}$.

Calame Blaise \& Calame-Griaule Geneviève (recueillies par), 1956, Scènes de la vie des Dogon, Résonances, Les Trésors de l'art musical populaire, $\mathrm{n}^{\circ} 9$.

Calame-Griaule Geneviève, 1970, « Pour une étude ethnolinguistique des littératures orales africaines » in POTTIER Bernard (dir.), Langages, $\mathrm{n}^{\circ} 18$, p. 22-47, DOI : 10.3406/lgge.1970.2026.

Calame-Griaule Geneviève, 1990, « "Prends ta houe mon frère". Chants funéraires dogon $\gg$ in Cahiers de littérature orale, $\mathrm{n}^{\circ} 27, \mathrm{p} .77-88$.

Calame-Griaule Geneviève, 2009, Ethnologie et langage. La parole chez les Dogon, $1^{\text {ere }}$ édition 1965, Lambert-Lucas, Limoges, 600 p.

CAnu Gaston, 1976, La langue mò:rē : dialecte de Ouagadougou, Haute-Volta : description synchronique, SELAF, Paris, France, 421 p.

Degorce Alice, 2012, «Un tipus d'enunciació en context ritual: els cants dels funerals moose (Burkina Faso)» in Quaderns-e de l'institut català d'antropologia (ICA), vol. 28, p. 55-73.

Degorce Alice (dir.), 2014, Chants funéraires des Mossi (Burkina Faso), Association classiques africains, Paris, 318 p.

Degorce Alice, 2016a, «Approches ethnolinguistiques des rapports entre hommes et femmes. Un objet en filigrane dans les recherches sur la parole en Afrique » in Lafay Marina, Le Guennec-Coppens Françoise \& Coulibaly Élisée (dir.), Regards scientifiques sur l'Afrique depuis les Indépendances, Éditions Karthala, Paris, France, p. 124-138.

Degorce Alice, 2016b, « Chanter dans les funérailles moose (Burkina Faso). Performances et pratiques vocales entre ritualité, deuil et créativité » in Poulet Charlotte \& BénARd Nicolas (dir.), Chant pensé, chant vécu, temps chanté : formes, usages et représentations des pratiques vocales, Éditions Delatour, Sampzon, p. 305-321. 
Fainzang Sylvie, 1985, « Les Sexes et leurs nombres. Sens et fonction du 3 et du 4 dans une société burkinabé » in L'Homme, n 96, p. 97-109, DOI : 10.3406/hom.1985.368624.

Gumperz John Joseph \& Hymes Dell, 1964, “The Ethnography of Communication" in The American Anthropologist, n ${ }^{\circ} 66$, pp. 1-29.

Hymes Dell, 1962, “The Ethnography of Speaking” in Gladwin Thomas \& Sturtevant William (eds.), Anthropology and Human Behavior, The Anthropological Society of Washington, Washington D.C., pp. 13-53.

Kapchan Deborah A., 1995, "Performance" in The Journal of American Folklore, $n^{\circ} 430$, vol. 108, pp. 479-508, DOI : $10.2307 / 541657$.

KawADA Junzô, 1998, La voix : étude d'ethno-linguistique comparative, Éditions de l'EHESS, Paris, 253 p.

Labov William, 1976, Sociolinguistique, trad. Kıнm Alain, Les Éditions de Minuit, Paris, 458 p.

Liberski Danouta, 1989, « Présentation » in Le Deuil et ses rites I. Systèmes de pensée en Afrique noire, n 9, p. 7-13.

Mauss Marcel, 1968, «L'expression obligatoire des sentiments » in Essais de sociologie, $1^{\text {ère }}$ édition 1921, Éditions de Minuit, Paris, p. 81-88.

Nikièma Norbert \& Kinda Jules, 1997, Moor gom-biis no-tũur gulsg sebre [Dictionnaire orthographique du mooré], Sous-commission nationale du mooré, Ouagadougou, $1316+120$ p.

Zongo Bernard, 2004, Parlons mooré : langue et culture des mossis, Burkina Faso, Paris, $215 \mathrm{p}$. 
Résumé : les chants funéraires constituent un domaine de l'oralité africaine souvent considéré comme peu accessible. Sans occulter la question du sens de ces chants, l'ethnolinguistique et l'anthropologie linguistique permettent d'aborder leur étude sous un angle privilégiant l'analyse du contexte d'énonciation et de la situation de performance. Cet article interroge ainsi des chants funéraires des Moose du Burkina Faso, à partir des travaux de Geneviève Calame-Griaule et d'un regard réflexif sur les questions méthodologiques posées par l'étude de ce corpus. Une analyse tenant compte de l'histoire locale, de la situation de performance, des réseaux de relations et du déroulement du rituel permet d'aborder les chants comme faisant partie intégrante du processus funéraire.

Mots-clefs: Chants, funérailles, rite, réflexivité, Mossi, Moose, Burkina Faso, Anthropologie.

\section{Studying Moose Funeral Songs}

Abstract: funeral songs are often considered as a field of orality whose meaning is difficult to access. Without leaving out this aspect, ethnolinguistic and linguistic anthropology allow one to address their study, giving priority to the analysis of contexts of enunciation and of performances. This paper examines in this way funeral songs of Moose people in Burkina Faso, with a thought based on Genevievve Calame-Griaule's work and on a reflexive look on methodological questions coming up with the study of this corpus. An analysis of local history, performance, relational networks and ritual proceedings allows considering theses songs as being part the funeral process.

Keywords: Songs, funeral, rite, reflexivity, Mossi, Moose, Burkina Faso, Social Anthropology.

\section{Moor kvı yuılg vausẽ n gese}

Koe-zugu : Kvya yull nĩnsabls zãmaane $n$ wã, yaa rog $n$ mik bümb sẽn pa tõe $n$ bãng nana ye. Buuda bãngr sẽn tüud ne buuda goam karẽngo, buuda sẽn yalé sẽn tuud ne buuda karẽng yaa soy sẽn tüund $n$ tõe $n$ karêm yulla sẽn yund wakat

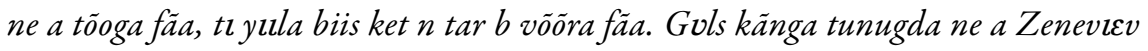
Kalam-Giriyol (Geneviève Calame-Griaule) tvoma, $n$ na $n$ soka mẽng $n$ gese, Burkina Faso kvya yul tõe $n$ tũu so bog $f$ sẽn dat $n$ wegsa $n$ kẽesa bãngr poagẽ. Karem-n-wegs sẽn tüud ne têmsa rog-n-miki, ne têmsa tõog buud fãa, neb ne taab 
CAHIERS DE LITTÉRATURE ORALE

126 Geneviève Calame-Griaule $-n^{\circ} 83$

bãngre, $n$ le paas kvora yel-pogds buud fãa kutdame tı kvora yull tõe $n$ dik wala bümb sẽn kẽed kvora büm-pogds poagẽ. ${ }^{44}$

Gom-bi : yula, kvvre, rog-n-miki, Moose, Burkina Faso.

\section{Note sur l'auteur}

Alice Degorce est chargée de recherche à l'IRD, elle a publié en 2014 Chants funéraires des Mossi dans la collection Classiques Africains. Elle poursuit actuellement ses travaux sur les paroles et les pratiques langagières associées aux migrations, aux rapports de genre et à la religion en Afrique de l'Ouest.

44. Nous remercions vivement Alain Ouédraogo, linguiste, chargé de recherche à l'Institut des Sciences des Sociétés (INSS/CNRST) de Ouagadougou, qui a traduit et transcrit le résumé et les mots-clés de cet article. 